NASA CONTRACTOR REPOR T

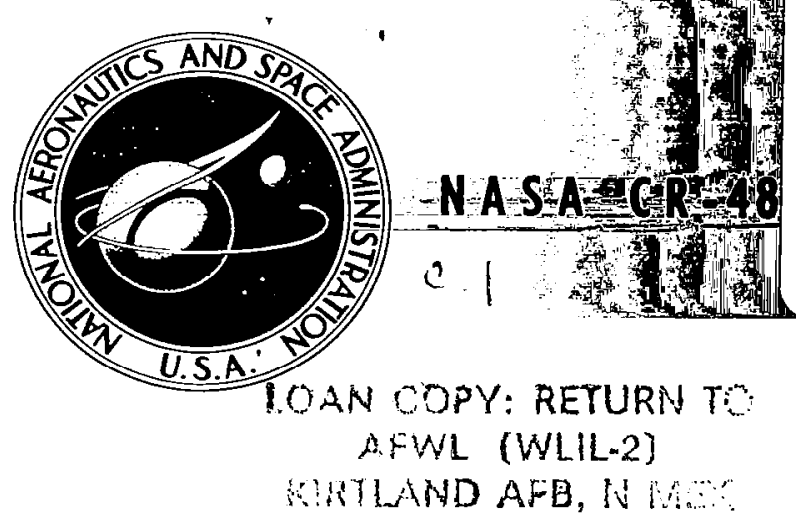

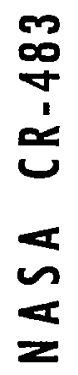

\title{
A DIRECT METHOD FOR CALCULATION \\ OF THE FLOW ABOUT AN AXISYMMETRIC BLUNT BODY AT ANGLE OF ATTACK
}

by Ibor O. Bobachevsky and Robert E. Mates

\section{Prepared by}

CORNELL AERONAUTICAL LABORATORY, INC.

Buffalo, N. Y.

for Goddard Space Flight Center

National aeronautics and SPACE Administration - Washington, D. C. • MaY 1966 


\section{A DIRECT METHOD FOR CALCULATION OF THE FLOW ABOUT}

\section{AN AXISYMMETRIC BLUNT BODY AT ANGLE OF ATTACK}

By Ihor O. Bohachevsky and Robert E. Mates

Distribution of this report is provided in the interest of information exchange. Responsibility for the contents resides in the author or organization that prepared it.

Prepared under Contract No. NAS 5-3976 by CORNELL AERONAUTICAL LABORATORY, INC. Buffalo, N.Y.

for Goddard Space Flight Center

NATIONAL AERONAUTICS AND SPACE ADMINISTRATION

For sale by the Clearinghouse for Federal Scientific and Technical Information Springfield, Virginia 22151 - Price $\$ 2.00$ 


\begin{abstract}
This report presents a solution for the supersonic inviscid flow around a spherically blunted body with a conically reentrant afterbody flying at a large angle of attack. The investigation was carried out by means of a direct numerical method for the calculation of unsteady, three-dimensional flow fields containing strong shock waves. The results presented include the location and shape of the bow shock and sonic lines, streamline patterns, the location of the stagnation point, and typical fluid property variations. The structure of the flow field is shown to be strongly influenced by the large angle of attack and by the body shape. The solution obtained is for an ideal gas; however, the method was developed in such a way that it can be readily extended to include nonequilibrium real gas effects. A description of the method and of special computational techniques required for its application is included.
\end{abstract}


TABLE OF CONTENTS

$\underline{\text { Section }}$

Page

ABSTRACT ......................... $i$

NOMENCLATURE ............... iv

LIST OF ILLUSTRATIONS. . . . . . . . . . . . v

1

INTRODUCTION ....................... 1

2

THE DIFFERENTIAL EQUATIONS . . . . . . . . 3

INITIAL AND BOUNDARY CONDITIONS . . . . . . . 4

4

THE DIFFERENCE EQUATIONS .......... . . 6

5

COMPUTATIONAL PROCEDURE ............ . 9

6 PRESENTATION OF RESULTS ............ 10

CONCLUDING REMARKS . . . . . . . . . . 14

REFERENCES . . . . . . . . . . . . . . . . 15 


\section{NOMENCLATURE}

$\begin{array}{ll}a & =\text { speed of sound, } \mathrm{cm} / \mathrm{sec} \\ f & =\text { general function of the flow variables } \\ h & =\text { enthalpy, ergs } / \mathrm{g} \\ p & =\text { pressure, dynes } / \mathrm{cm}^{2} \\ g & =\text { particle speed, } \mathrm{cm} / \mathrm{sec} \\ r & =\text { radius, } \mathrm{cm} \\ t & =\text { time, sec } \\ u & =\text { radial velocity component, cm } / \mathrm{sec} \\ v & =\text { angular }(\theta) \text { velocity component, } \mathrm{cm} / \mathrm{sec} \\ \omega & =\text { aximuthal }(\varphi) \text { velocity component, } \mathrm{cm} / \mathrm{sec} \\ \alpha & =\text { angle of attack } \\ \gamma & =\text { adiabatic exponent } \\ \theta, \varphi & =\text { angular coordinates } \\ \rho & =\text { density, } \mathrm{g} / \mathrm{cm}^{3}\end{array}$

Subscripts

b $=$ on the surface of the body

$j, k=\theta$ and $\varphi$ position in the mesh

$0 \quad=$ free-stream (initial) values

$t, \Omega, \theta, \varphi=$ partial derivatives

Superscript

$i=$ radial position in the mesh 


\section{LIST OF ILLUSTRATIONS}

Figure

Page

Coordinate System . . . . . . . . . . . . . . 17

2

Computing Region . . . . . . . . . . . . . . . 18

Radial Density Distribution . . . . . . . . . . . . 19

Shock Shape in the Plane of Symmetry . . . . . . . . . . 20

Radial Density Distribution . . . . . . . . . . . . . 21

Surface Pressure Distribution in the Plane of Symmetry . 22

Streamline Pattern in the Plane of Symmetry . . . . . . 23

The Stagnation Region. . . . . . . . . . . . . . . . 24

9 Shock and Sonic Line For Symmetric Flow . . . . . . . . 25

10 Shock and Sonic Line for Symmetric Flow . . . . . . . . 26

1. 1 Streamline Pattern on the Spherical Foŕebody Surface . 27

12 Streamline Pressure Distribution . . . . . . . . . . . 28 


\section{INTRODUCTION}

The purpose of this work was to determine the structure of the inviscid flow field developed in front of a blunt body with corners in supersonic flight at a large angle of attack. The work was motivated by the proposed use of maneuverable, lifting reentry vehicles for lunar and interplanetary missions. Therefore, the shape selected for this investigation is a spherically blunted forebody with a conically reentrant afterbody (see Fig. 1) which closely resembles the APOLLO vehicle.

For the above configuration, the flow field is described by means of an exact numerical solution of the governing differential equations. The results obtained include the location and shape of the shock wave and sonic lines, the surface pressure distribution, streamline patterns on the body surface and in the plane of symmetry, and typical fluid property variations. They indicate that the flow field is strongly asymmetric. The theoretical solution was compared with the available experimental data and the agreement found to be satisfactory.

The problem of describing the shock layer in front of a blunt body has received considerable attention. The primary difficulty arises because of an incomplete knowledge of the boundary conditions on the shock layer; the location and strength of the bow shock and the location of the sonic line are not known. Several methods have been proposed for circumventing this difficulty; however, most of these techniques rely heavily on assumptions of axisymmetric flow and/or a smooth body contour.

The inverse method begins with an assumed shock shape and determines the corresponding body. Van Dyke ${ }^{1}$ and Garabedian and Lieberstein ${ }^{2}$ originally applied the technique to axisymmetric flows about bodies with smooth contours. Since, in the inverse problem, the body shape obtainedis extremely sensitive to the assumed shock shape, the procedure is unsuitable for contours with sharp corners. For the same reason, the method is difficult to apply to unsymmetric flow fields, since the required shock shape is complex. So far, the inverse technique has been applied successfully to 
asymmetric flow fields when the angle of attack is sufficiently small to introduce only a perturbation on the symmetric solution. 3,4

The direct method of integral relations ${ }^{5}$ has also been applied to asymmetric flows by several authors. $6,7,8$ In such application, some additional assumption must be made: either that the maximum entropy streamline wets the body ${ }^{6,7}$ or that the stagnation streamline is perpendicular to the body surface. ${ }^{8}$ The present work, as well as the work of Swigart, ${ }^{3}$ indicates that this is, in general, not true. In addition, this method becomes unwieldy when better than one strip approximation is attempted.

The difficulty of unknown boundary conditions can be avoided by solving the unsteady problem for which the governing equations are hyperbolic. The body is accelerated from rest and the asymptotic (for large time) behavior of the unsteady flow field is determined. This is the technique employed in the present work. This procedure was originally used by Godunov and coworkers; ${ }^{9}$ however, the application of their elaborate differencing scheme to three-dimensional flows appears impractical.

Hence, in the present work, the method suggested by Bohachevsky and Rubin ${ }^{10}$ for the computation of supersonic flows with detached shock waves was followed. In the computational process, a generalization of the finite difference scheme first proposed by Lax ${ }^{11}$ was employed. The simplicity of this scheme, which is discussed in Sections 1 to 4, was a major factor in the success of the present work. Application of the method, however, necessitated the development of special computing techniques because the storage capacity of the available IBM 7044 computer was insufficient for the large amount of data required by the three-dimensional flow field. This difficulty was circumvented by using magnetic tapes for auxiliary storage. To keep the computing time at reasonable length, the tapes were read sequentially and not used as a random access memory. 
2. THE DIFFERENTIAL EQUATIONS

Most difficulties in treating initial-boundary value problems are encountered, not in solving the differential equations, but in attempting to satisfy the boundary conditions. Therefore, a body-oriented coordinate system is adopted to simplify the latter task. For a spherically blunted body, the spherical polar coordinates are appropriate. The use of this coordinate system (Fig. 1) requires two modifications (which will be introdiced later) in the computing procedure, but the ease with which the boundary conditions can be satisfied far outweighs the resulting complications.

It was shown originally by $\operatorname{Lax}^{11}$ that numerical solutions for flow fields containing strong shocks may be obtained if the governing equations are written in conservation, or divergence, form. In spherical coordinates, this form of the inviscid equation is:

a. Conservation of Mass

$$
(\rho)_{t} r^{2} \sin \theta+\left(\rho u r^{2} \sin \theta\right)_{\Omega}+(\rho v r \sin \theta)_{\theta}+(\rho \omega r)_{\varphi}=0
$$

b. Conservation of the Three Momentum Components

$$
\begin{aligned}
& (\rho u)_{t} r^{2} \sin \theta+\left[\left(\rho u^{2}+p\right) r^{2} \sin \theta\right]_{n}+(\rho u v r \sin \theta)_{\theta}+ \\
& (\rho u w r)_{p}=\left[2 p+\rho\left(N^{2}+w^{2}\right)\right] r \sin \theta \\
& (\rho v)_{t} r^{2} \sin \theta+\left(\rho u v r^{2} \sin \theta\right)_{r}+\left[\left(\rho r^{2}+p\right) r \sin \theta\right]_{\theta}+ \\
& (\rho v \omega r)_{p}=\left(p+\rho \omega^{2}\right) x \cos \theta-\rho u v r \sin \theta \\
& (\rho w)_{t} r^{2} \sin \theta+\left(\rho \mu w r^{2} \sin \theta\right)_{n}+(\rho v w r \sin \theta)_{0}+ \\
& {\left[\left(\rho \omega^{2}+p\right) r\right]_{\varphi}=-\rho \mu \omega r \sin \theta-\rho N \omega r \cos \theta}
\end{aligned}
$$

3 
c. Conservation of Energy

$$
\begin{aligned}
&\left(\frac{1}{2} \rho g^{2}+\rho h-p\right)_{t} r^{2} \sin \theta+\left[\rho \mu\left(\frac{1}{2} g^{2}+h\right) r^{2} \sin \theta\right]_{\pi}+ \\
& {\left[\rho N\left(\frac{1}{2} g^{2}+h\right) r \sin \theta\right]_{\theta}+\left[\rho \omega\left(\frac{1}{2} g^{2}+h\right) \pi\right]_{\varphi}=0 }
\end{aligned}
$$

where $u$ is the radial, $v$ the angular $(\theta)$, w the aximuthal $(\varphi)$ component of velocity, $q^{2}=u^{2}+v^{2}+w^{2}, \rho$ is the density, $p$ is the pressure, and $h$ is the enthalpy per unit mass.

Equations (1) to (5) must be supplemented with a thermodynamic relation

$$
h=\frac{\gamma}{\gamma-1} \frac{p}{\rho}
$$

where $\gamma$ is the ratio of the specific heats.

\section{INITIAL AND BOUNDARY CONDITIONS}

We shall seek that solution of Equations (1) to (6) which, at $t=0$ satis fies the following initial conditions

$$
\begin{aligned}
& \rho=p_{0}=\text { constant } \\
& u=u_{0}=-q_{0}(\cos \alpha \cos \theta+\sin \alpha \sin \theta \cos \varphi) \\
& v=v_{0}=q_{0}(\cos \alpha \sin \theta-\sin \alpha \cos \theta \cos \varphi) \\
& w=w_{0}=q_{0} \sin \alpha \sin \varphi \\
& p=p_{0}=\text { constant }
\end{aligned}
$$

The above conditions represent a free stream of constant speed $q_{0}$ at the angle $\propto$ with respect to the ray $\theta=0$ which is the axis of symmetry of the body. Figure 1 illustrates the geometry of the problem.

The only boundary condition which should be imposed on the solution is the vanishing of the normal velocity component at the surface of the body. 
Referring to Figure 1, this condition in the present case can be expressed as follows:

a. On $r=r_{b}\left(0 \leq \theta \leq \theta_{b}\right) \quad u=0$

b. On $\theta=\theta_{b}\left(\kappa \leq r_{b}\right) \quad w=0$

c. On $\varphi=0$ and $\pi$, which is the plane of symmetry formed by the velocity vector and the axis of the body, $\omega=0$.

At $\theta=0$, which is the singular point of the coordinate system but a regular, interior point of the flow field, Equations (1) through (5) are not applicable. They are replaced by the following set

$$
(\rho)_{t} n^{2}+\left(\rho u r^{2}\right)_{n}+2(\rho \sim r)_{\theta}+(\rho w r)_{\varphi \theta}=0
$$

$$
\begin{aligned}
(\rho u)_{t} t^{2}+\left[r^{2}\left(\rho u^{2}+p\right)\right]_{n}+ & 2(\rho u \omega r)_{\theta}+(\rho u \omega r)_{\varphi \theta}= \\
& {\left[2 \rho+\rho\left(\omega^{2}+\omega^{2}\right)\right] \tau }
\end{aligned}
$$

$$
\begin{aligned}
(\rho v)_{t} x^{2}+\left(\rho \mu w r^{2}\right)_{r}+ & {\left[r\left(2 \rho v^{2}+p\right)\right]_{\theta}+(\rho w \mu r)_{\varphi \theta}=} \\
& \left(\rho w^{2} r\right)_{\theta}-\rho u v r
\end{aligned}
$$

$$
(\rho w)_{t} n^{2}+\left(\rho \mu w r^{2}\right)_{n}+3(\rho w w r)_{\theta}+\left[r\left(\rho w^{2}+p\right)\right]_{\varphi \theta}=
$$

$-\rho u \mu \kappa$

$$
\begin{aligned}
r^{2}\left(\frac{1}{2} \rho q^{2}+\rho h-p\right)_{t}+ & {\left[\rho u r^{2}\left(\frac{1}{2} g^{2}+h\right)\right]_{\Omega}+2\left[\rho \sim r\left(\frac{1}{2} g^{2}+h\right)\right]_{0}+} \\
& {\left[\rho \operatorname{wrr}\left(\frac{1}{2} g^{2}+h\right)\right]_{\varphi \theta}=0 }
\end{aligned}
$$

5 
The above relations are obtained by dividing each of the Equations (1) to (5) by $\sin \theta$ and evaluating the limit as $\theta \rightarrow 0$, remembering that at $\theta=0$, $\frac{\partial}{\partial \varphi}=0$. This is the first of the two required modifications mentioned at the beginning of Section 1 .

4. THE DIFFERENCE EQUATIONS

For the purpose of digital computation, the differential Equations (1) to (5) (and (7) to (11)) are replaced by the difference equations obtained in the following way

$$
\begin{aligned}
& f(r, \theta, \varphi, t) \longrightarrow \frac{1}{2 \Delta n}\left[f_{j, k}^{i}(t)\right. \\
& \left.f_{k}^{i+1}(t)-f_{j, k}^{i-1}(t)\right] \\
& f_{\theta} \longrightarrow \frac{1}{2 \Delta \theta}\left[f_{j+1, k}^{i}(t)-f_{j-1, k}^{i}(t)\right] \\
& f_{\varphi} \longrightarrow \frac{1}{2 \Delta \varphi}\left[f_{j, k+1}^{i}(t)-f_{j, k-1}^{i}(t)\right]
\end{aligned}
$$

Note that, in this process, each group of terms must not be separated, i.e., the product rule for differentiation should not be used.

The time differences must be treated with more care. An averaging process must be carried out in order to prevent divergence at the shock and the averaging must be so performed that the free stream remains essentially constant in time. Since the velocity components $(u, w, w)$ in the free stream are dependent on the location of the mesh points, simple averaging will cause a time variation of the free -stream velocity. This variation must be corrected in the averaging process. With these considerations in mind, the time derivetives are replaced by the following differences:

$$
\begin{gathered}
f_{t} \rightarrow \frac{1}{\Delta t}\left\{f_{j, k}^{i}(t+\Delta t)-\frac{1}{6}\left[f_{j, k}^{i+1}(t)+f_{j, k}^{i-1}(t)+f_{j+1, k}^{i}(t)+\right.\right. \\
\left.\left.f_{j-1, k}^{i}(t)+f_{j, k+1}^{i}(t)+f_{j, k-1}^{i}(t)\right]\right\}
\end{gathered}
$$

6 
where $f$ is $\rho$ or $\frac{1}{2} \rho q^{2}+\rho h-p$ and

$$
\begin{aligned}
& (\rho u)_{t} \rightarrow \frac{1}{\Delta t}\left\{(\rho u)_{j, k}^{i}(t+\Delta t)-\frac{1}{6}\left[(\rho u)_{j, k}^{i+1}(t)+\rho u_{j, k}^{i-1}(t)+\right.\right. \\
& (\rho u)_{j+1, k}^{i}(t)+(\rho u)_{j-1, k}^{i}(t)+(\rho u)_{j, k+1}^{i}(t)+ \\
& (p u)_{j, t-1}^{i}(t)+(\Delta \theta)^{2}(p u)_{j, k}^{i}(t)+ \\
& \left.\left.(\Delta \varphi)^{2}(\rho g \sin \alpha \sin \theta \cos \varphi)_{j, k}^{i}(t)\right]\right\} \\
& (\rho \sim)_{t} \longrightarrow \frac{1}{\Delta t}\left\{(\rho v)_{j, k}^{i}(t+\Delta t)-\frac{1}{6}\left[(\rho \sim)_{j, k}^{i+1}(t)+(\rho \sim)_{j, k}^{i-1}(t)+\right.\right. \\
& (p \sim)_{j+1, k}^{i}(t)+(p \sim)_{j-1, k}^{i}(t)+(p w)_{j, k+1}^{i}(t)+ \\
& (\rho \sim)_{j, k-1}^{i}(t)+(\Delta \theta)^{2}(\rho \sim)_{j, k}^{i}(t)- \\
& \left.\left.(\Delta \varphi)^{2}(\rho q \sin \alpha \cos \theta \cos \varphi)_{j, k}^{i}(t)\right]\right\} \\
& (\rho w)_{t} \longrightarrow \frac{1}{\Delta t}\left\{(\rho \omega)_{j, k}^{i}(t+\Delta t)-\frac{1}{6}\left[(\rho \omega)_{j, k}^{i+1}(t)+(\rho \omega)_{j, k}^{i-1}(t)+\right.\right. \\
& (\rho \omega)_{j+1, k}^{i}(t)+(\rho w)_{j-1, k}^{i}(t)+(\rho w)_{j, k+1}^{i}(t)+ \\
& \left.\left.(\rho w)_{j, k-1}^{i}(t)+(\Delta \varphi)^{2}(\rho w)_{j, k}^{i}(t)\right]\right\}
\end{aligned}
$$

This difference scheme has been discussed in detail in Reference 10 and in references cited there. The change of averaging in the time differences of the velocity components is the second modification mentioned previously.

The boundary conditions for the entire computing region shown in Figure 2 are specified as discussed in Reference 10 in the following way: the grid is extended one mesh width inside the solid body and outside the region of computation. None of the variables is computed there but assigned a value according to the following rules:

7 
(a) $u$ at $\kappa_{b}-\Delta r$ for $\theta \leqslant \theta_{b}$ is the negative of $u$ at $\kappa_{b}+\Delta n$; all other variables assume at $\kappa_{b}-\Delta n$ the same values they have at $r_{6}+\Delta r$.

(b) $\sim$ at $\theta_{b}-\Delta \theta$ for $\tau \leqslant n_{b}$ is the negative of $\sim$ at $\theta_{b}+\Delta \theta$; all other variables assume at $\theta_{b}-\Delta \theta$ the same values they have at $\theta_{b}+\Delta \theta$.

(c) $\quad \omega$ at $-\Delta \varphi$ and $\pi+\Delta \varphi$ is the negative of $\omega$ at $\Delta \varphi$ and $\pi-\Delta \varphi$, respectively; other variables are to have the same values at points reflected in the $\varphi=0$ and $\varphi=\pi$ plane.

(d) At the outer boundary of the mesh in the $\theta$ direction $\theta_{\max }$ for $r_{\min } \leq n \leq r_{\max }$ and at the inner boundary of the mesh in the radial direction $n_{m i n}$ for $\theta_{b} \leqslant \theta \leqslant \theta_{\max }$ we prescribe the vanishing of the second difference, i.e., use a linear extrapolation procedure.

(e) At the outer boundary of the mesh in the radial direction, denoted by $\Lambda_{\max }$ for $0 \leq \theta \leq \theta_{\max }$, we hold all the variables equal to their free-stream values. It should be observed here that these values of $\rho_{0}, g_{0}$ and $p_{0}$ need not be constants, but may vary with time in a prescribed manner. The solution will then represent flight through a nonuniform atmosphere, in which case the steady state will never be reached but the method remains applicable. This property may also be used to generate families of solutions by changing free-stream Mach number or angle of attack and attaining the steady state for each new value. Such procedure will result in significantly more economical use of the computer.

(f) At the corner point of the body (A in Fig. 2) when computing (updating) the values of the flow variables at $B$, the necessary values at $D$ are obtained as in (a) above; when computing at $C$ the necessary values are obtained as in (b). Thus, $u$ and $v$ (and other variables, if necessary) are double-valued at $D$, the value used depending on where the computation is being performed. 
In order to obtain satisfactory results, it is necessary that the surface $r=n_{\max }$ lie in the undisturbed free stream (i.e., well ahead of the bow shock) and that $\theta=\theta_{\text {max }}$ together with $\pi=\kappa_{\min }$ lie wholly in the supersonic region of the flow. It has been established in Reference 10 that when these requirements are met, the conditions prescribed at those surfaces do not affect the solution.

\section{COMPUTATIONAL PROCEDURE}

The solution of the difference equations is straightforward. The values of the flow quantities are advanced in time using the finite difference analogues of Equations (1) to (5) successively in the same order in which they are written. The ensuing simplicity of the program logic makes it relatively easy to apply the method to flows about complex geometries and to extend the method to include nonequilibrium effects.

The magnitude of the time step $\Delta t$ used in the advance is controlled by the stability requirement. The Courant-Friedrichs-Lewy stability conditions which apply to linear hyperbolic partial differential equations are

$$
\frac{\Delta t}{\Delta r} \leqslant \frac{1}{|u|+a} ; \quad \frac{\Delta t}{n \Delta \theta} \leq \frac{1}{|v|+a} ; \quad \frac{\Delta t}{r \sin \theta \Delta \varphi} \leq \frac{1}{|\omega|+a}
$$

where $a$ is the speed of sound. The computations reported in Reference 10 suggest that these conditions are sufficient; in his investigation of the onedimensional flows, Lax ${ }^{11}$ points out that they appear necessary. We used them throughout the nonlinear field by estimating the smallest value of the right-hand side of the inequalities and choosing $\Delta t$ accordingly and experienced no difficulty. Since the ray $\theta=0$ is treated in a special manner, the vanishing of $\sin \theta$ does not cause any trouble.

To obtain best results, $\Delta t$ should have the largest possible value which permits a stable computation since the spread of the shock transition is proportional primarily to $(\Delta r)^{2} / \Delta t$. For the mesh size which we used, this value of $\Delta t$ was $3.5 \times 10^{-6}$ sec. 
The primary difficulty in obtaining a solution stems from the number of space points required for an adequate definition of the flow field. For the computations presented in this paper the body shape was characterized (referring to Fig. 1) by $r_{b}=474 \mathrm{~cm}$ and $\theta_{b}=35^{\circ}$. The computing region in one meridian plane is depicted in Fig. 2 where $r_{\text {max }}=664 \mathrm{~cm}$, $\theta_{\text {max }} \approx 2 \theta_{b}$, and $r_{\text {min }}=r_{b}-10 \Delta r$. In the complete description of the three-dimensional field we used 30 such meridian planes. For the mesh size which we employed, i. e., $\Delta r=5 \mathrm{~cm}, \Delta \theta=.068, \Delta \varphi=\pi / 30$, the field contained about 24,000 points. Even when the solution is restricted to the case of an ideal gas whose flow can be described by five variables, the number of values which must be stored is about 120,000. This difficulty was circumvented by recording the flow variables on the magnetic tape. The use of magnetic tape storage does not increase the calculation times excessively since the tape is not used as a random access memory.

\section{PRESENTATION OF RESULTS}

In this section are presented the results of digital computations for an APOLLO-shaped vehicle at an angle of attack of $20^{\circ}$. The initial (free stream) conditions were $q_{0}=9.16 \times 10^{5} \mathrm{~cm} / \mathrm{sec}, \rho_{0}=3.14 \times 10^{-7} \mathrm{~g} / \mathrm{m}^{3}$, $p_{0}=2.26 \times 10^{2}$ dynes $/ \mathrm{cm}^{2}, \alpha=20^{\circ}$ which for the 1959 ARDC atmosphere correspond to Mach number 28.9 (velocity $=30,000 \mathrm{ft} / \mathrm{sec}$ ) at an altitude of $200,000 \mathrm{ft}$ in air for which $\gamma=1.4$.

Because of the averaging in the finite difference equations, the shock is spread out over a finite distance in the flow field, typically 4 or 5 mesh widths. It was found in Reference 10 by comparison with the inverse method, that the shock can be located accurately by assigning its position to the midpoint of the density rise. The radial density distribution for several values of $\theta$ in the plane $\varphi=0$ is shown in Figure 3.

Using these results and a similar plot for $\varphi=180^{\circ}$, the shock shape in the plane of symmetry can be determined. This shock shape is shown in 
Figure 4 for several values of time. Initially, the shock moves out nearly parallel to the surface; rotation to account for the angle of attack occurs near the end of the calculation. Because of this, it is expected that a solution for a different angle of attack could be obtained in a relatively short computing time using the present result as a starting point. Similar plots can be made for any meridian plane $\varphi=$ constant. For example, Figure 5 represents the radial density distribution in front of the body in the plane $\varphi=120^{\circ}$. Comparison of Figures 3 and 5 also illustrates the effect of mesh size on the thickness of the shock. The results in Figure 3 were obtained with a refined mesh in the radial direction of $2.5 \mathrm{~cm}$ and those in Figure 5 with a $5 \mathrm{~cm}$ mesh.

Figure 6 represents the surface pressure distribution for the plane of symmetry after 150 time steps which was the number of steps required to establish the steady state, i.e., when the flow variables no longer change appreciably with time. From a similar plot of the $\theta$ velocity component $\sim$ we obtain the location of the stagnation point at the value of $\theta$ where $v=0$ which for the present problem is $14.97^{\circ}$. This is in good agreement with the experimental value of $14.5^{\circ}$ given by Kaattari. ${ }^{2}$ The dotted curved indicates the simple Newtonian impact pressure distribution $p=\rho_{0} q_{0}^{2}\left[1-\sin ^{2}(\theta-\alpha)\right]$. The agreement is quite good on the leeward side, becomes poorer towards the stagnation point, and is worse on the windward side. The calculated pressure is less than the Newtonian value. Considering the approximate nature of the Newtonian theory, the agreement is surprisingly good in general.

The streamline pattern in the plane of symmetry is shown in Figures 7 and 8. Figure 7 represents the entire plane and Figure 8 is the enlarged view of the stagnation region. The streamlines themselves are obtained as integral curves of the direction field defined by the velocity vector (which is part of the numerical output) at each mesh point. In general, a streamline does not pass through the mesh points exactly and a linear interpolation is used to obtain its slope at intermediate points. The accuracy of such numerical integration of the velocity field was checked by using 
successively smaller steps. From these results, it is apparent that the maximum entropy streamline, which passes normally through the shock, does not wet the body.

Figure 7 includes also the sonic line which on the windward side reaches the body behind the corner. (In view of the boundary logic for the finite difference scheme in this work, the corner is not sharp but has a radius of curvature less than one mesh width.) In most aerodynamic analyses, $5,7,12-14$ it is usually assumed that on a body with sharp corners the sonic line will be attached to the corner. It appears reasonable, however, that with a sufficiently strong bow shock, the Mach number upstream of the corner will be so low that the expansion around the corner may be insufficient to cause transonic flow.

In the present result the distance from the corner to the sonic point, 7 radial mesh points, is a small fraction of the body radius (0.04). Because of the dissipation introduced with the averaging process, ${ }^{10}$ the flow along streamlines is not exactly isentropic; this influences the location of the sonic line to some extent. These facts indicate the need for additional evidence before a definite conclusion can be drawn.

A series of calculations was carried out for the zero angle of attack to determine the influence of the free stream Mach number and afterbody angle on the location of the sonic line. The shapes of the shock and sonic line for $\theta_{b}=35^{\circ}$ are shown in Figure 9 for free stream Mach numbers 29 and 4. The intersection of the sonic line with the body occurs in each case downstream of the corner and moves slightly further downstream with increasing free stream Mach number consistent with the lower value of the Mach number upstream of the corner. Since the distance from the corner to the sonic line attachment point is 12 to 13 radial mesh widths for these calculations this effect does not appear to be caused by the lack of resolution in the solution.

Corresponding results for $\theta_{b}=19.5^{\circ}$ at the free stream Mach number of 29 are shown in Figure 10. Here the sonic line attaches also behind the corner. Similar calculations were carried out for the body angle larger than the extent of the subsonic flow region on the sphere. As expected, the sonic 
line was attached to the body upstream of the corner and the results agreed with those reported in References 5 and 10.

To ensure that the observed sonic line position was not a transient phenomenon its location was monitored during the course of the computation. The position of the sonic line was established quite early in the calculation process and showed no tendency to move toward the corner as the steady state was approached asymptotically.

The small circles in Figure 7 denote the shock shape predicted by the empirical correlation presented in Reference 12. The shock layer thickness near the body axis agrees very well; however, the present calculations indicate a larger standoff distance near the corners. The experimental results for higher Mach numbers given by Kaattari in Reference 14 also show a larger standoff distance than the correlation near the corners and hence are consistent with our solution.

Figure 11, the projection of the surface streamline pattern onto the plane normal to the axis of the body, illustrates the direction of the flow field on the spherical forebody. The sonic line location is also shown.

The dashed lines in Figure 11 represent the Newtonian paths which are arcs of great circles passing through the stagnation point. The actual streamlines deviate considerably from the Newtonian prediction; because of the high pressure on the windward side the particles drift to leeward. This streamline torsion illustrates the three-dimensional nature of the solution and indicates the magnitude of the cross flow. These streamline patterns are in good agreement with the qualitative experimental results of Kaattari. 12

Simultaneously with the location of the streamlines, the numerical program computes the distribution of the thermodynamic variables along them. A typical result is shown in Figure 12 which represents the pressure distribution along streamline $A$ in Figure 7. The pressure rises abruptly through the shock, decreases gradually as the flow expands along the body and finally drops rapidly at the corner. The peculiar behavior of pressure just behind the shock is apparently due to the numerical method and does not have any physical significance. 


\section{CONCLUDING REMARKS}

The object of this work was to develop a direct numerical technique for the computation of the flow field around a blunt body in hypersonic flight at an angle of attack. A solution has been obtained for an APOLLOshaped vehicle at $20^{\circ}$ angle of attack.

The results indicate that the flow field is strongly three-dimensional. The stagnation point is located at a smaller angle from the body axis than is the free-stream velocity vector. The streamline which passes normally through the shock does not wet the body. The streamlines on the body surface show strong torsion which suggests that the boundary layer on the body will exhibit large cross-flow effects. There is evidence that the sonic line on the windward side of the body is not attached to the corner, but lies behind the corner. The results are in agreement with the available experimental data of Kaattari.

The solution presented was carried out for an ideal gas in order to demonstrate the feasibility of the method for a simple case. By replacing Equation (6) by the appropriate expressions for a multicomponent mixture and adding the equations of chemical kinetics, it is possible to include the effects of nonequilibrium dissociation and ionization on the flow field. This calculation is currently being carried out.

\section{ACKNOW LEDGMENT}

We wish to acknowledge many helpful discussions with our colleague, Dr. M. Dunn, concerning the presentation of these results, and the able assistance of $\mathrm{Mr}$. H. Rosenbaum, who wrote the original computer program, and Mr. J. Moselle, who carried out the computations. 


\section{REFERENCES}

1. Van Dyke, M., "The Supersonic Blunt-Body Problem - Review and Extensions," J. Aero. Sci. 25, 485-496 (1958).

2. Garabedian, P.R. and Lieberstein, H.M., "On the Numerical Calculation of Detached Bow Shock Waves in Hypersonic Flow, " J. Aero. Sci. 25, 109-118 (1958).

3. Swigart, R., "Hypersonic Blunt-Body Flow Fields at Angle of Attack," AIAA J. 2, 115-117 (1964).

4. Swenson, E.V., "Numerical Computation of Hypersonic Flow Past a Two-Dimensional Blunt Body," AEC Res. and Develop. Report NYO 1480-1, C.I.M.S., New York University (1964).

5. Bielotserkovskii, O.M., "On the Calculation of Flow Past Axisymmetric Bodies with Detached Shock Waves Using an Electronic Computing Machine," P. M. M. 24, $511-517$ (1960).

6. Prosnak, W.J. and Luczywek, E., "The Direct Asymmetric Hypersonic Blunt-Body Problem," AIAA Preprint 64-552.

7. Bazzhin, A.P., "On the Computation of the Supersonic Flow About a Flat Plate with a Detached Shock Wave," Inzh. Zh. $3,222-227$.

8. Waldman, G.D., "Integral Approach to the Yawed Blunt Body Problem," AIAA Paper 65-28.

9. Gudunov, S.K., Zabrodin, A.O., and Prokopov, G.P., "A Difference Scheme for Two-Dimensional Unsteady Flow with a Detached Shock Wave," Zh. Vychyslitelnoi Mat.i Mat. Fiziki I, 1020-1050 (1961); available as a CAL Translation.

10. Bohachevsky, I. O. and Rubin, E. L., "A Direct Method for Computation of Nonequilibrium Flows with Detached Shock Waves," AIAA J. (submitted for publication).

11. Lax, P.D., "Weak Solutions of Nonlinear Hyperbolic Equations and Their Numerical Computations," Communs. Pure and Appl. Math $\underline{7}$, $159-193$ (1954).

12. Kaattari, G. E., "Shock Envelopes of Blunt Bodies at Large Angles of Attack," NASA TN D-1980 (Dec. 1963). 
13. Vaglio-Laurin, R., "Transonic Rotational Flow Over a Convex Corner," J. Fluid Mech. 9, 81-103 (1960).

14. Kaattari, G. E., "Predicted Shock Envelopes About Two Types of Vehicles at Large Angles of Attack," NASA TN D-860 (April 1961). 

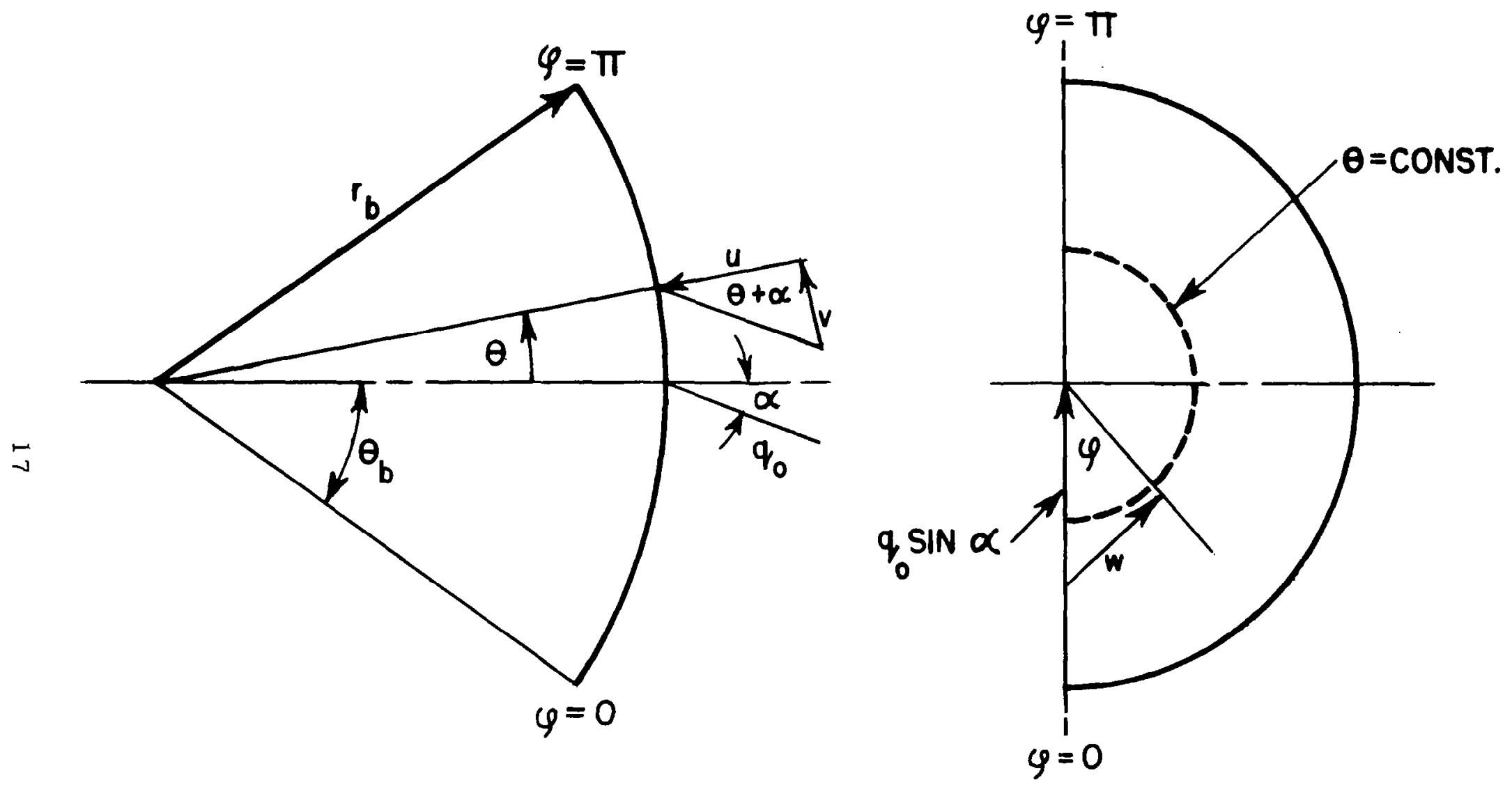

Figure I COORDINATE SYSTEM 


$$
\begin{aligned}
r_{\max } & =664 \mathrm{~cm} \\
r_{b} & =474 \mathrm{~cm} \\
r_{\min } & =424 \mathrm{~cm} \\
\theta_{b} & =35^{\circ} \\
\theta_{\max } & =62.3^{\circ}
\end{aligned}
$$

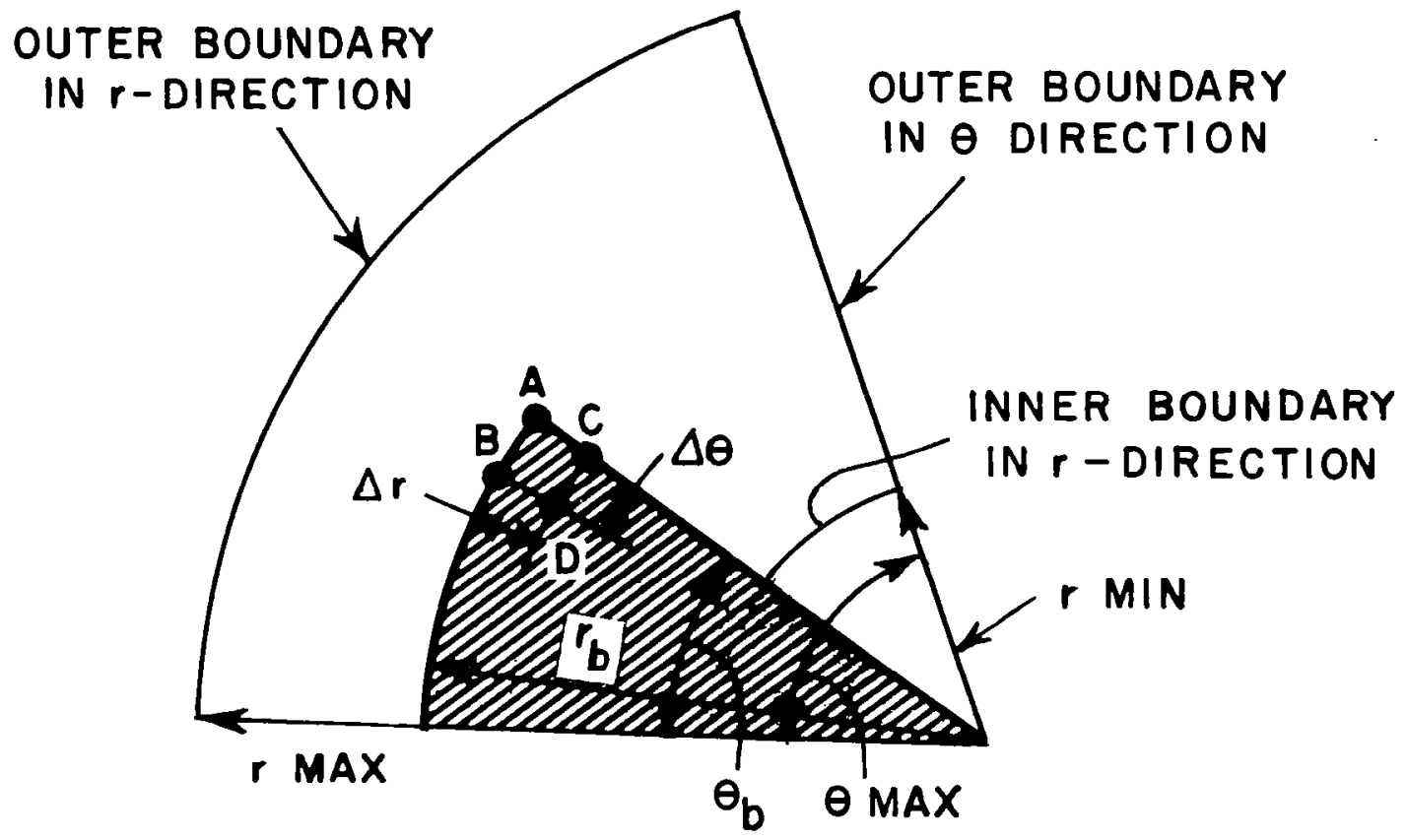

Figure 2 COMPUTING REGION 


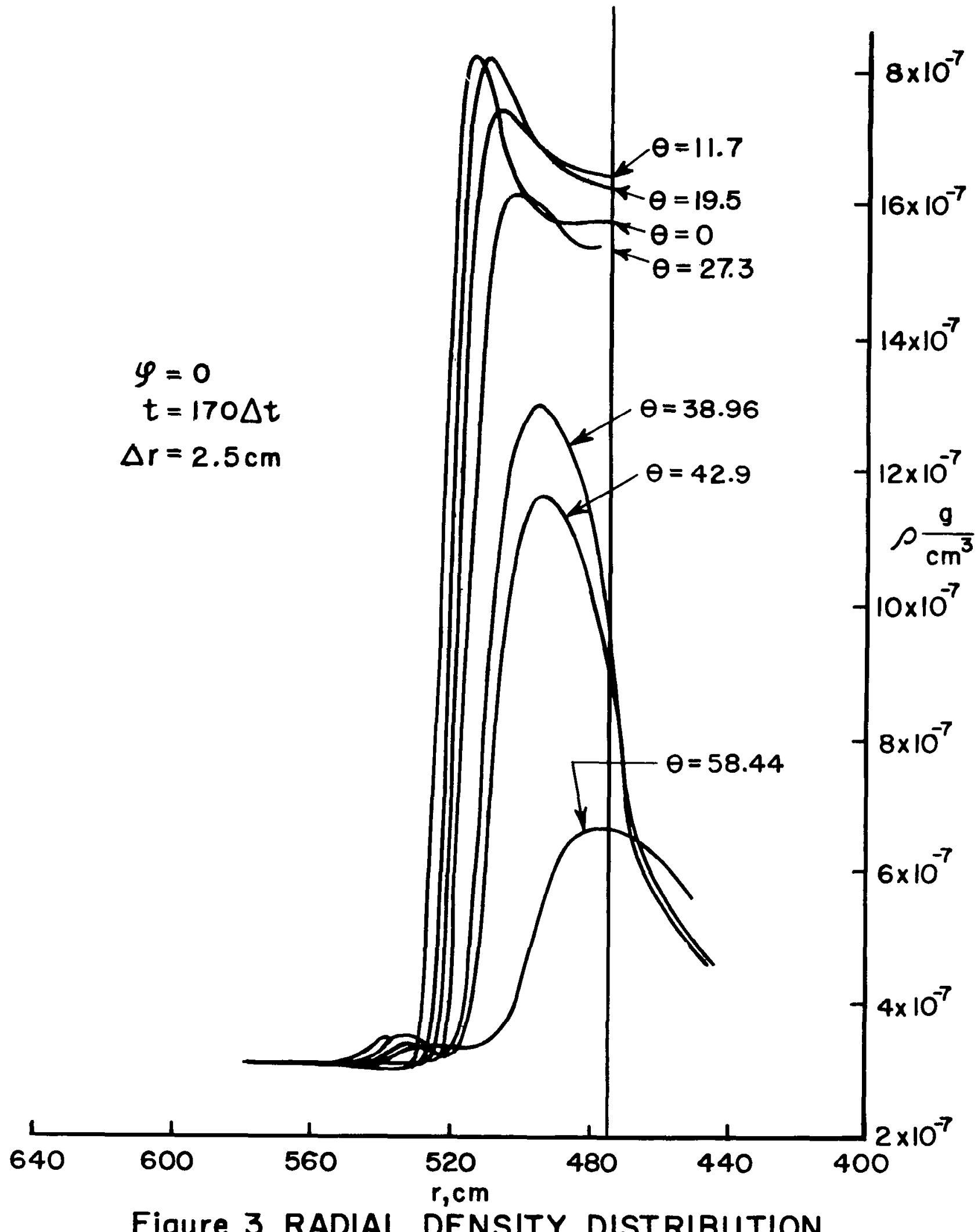

Figure 3 RADIAL DENSITY DISTRIBUTION 


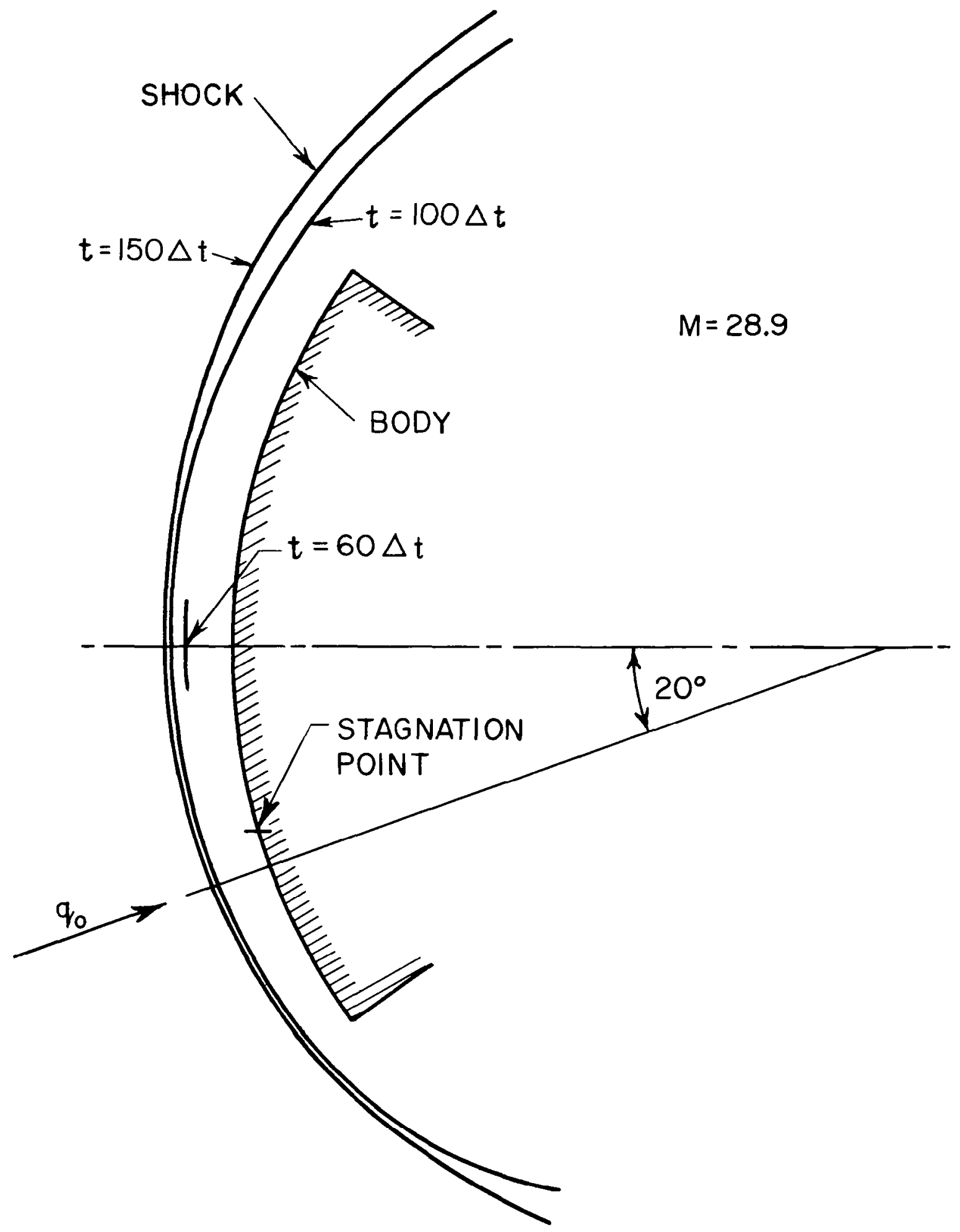

Figure 4 SHOCK SHAPE IN THE PLANE OF SYMMETRY 


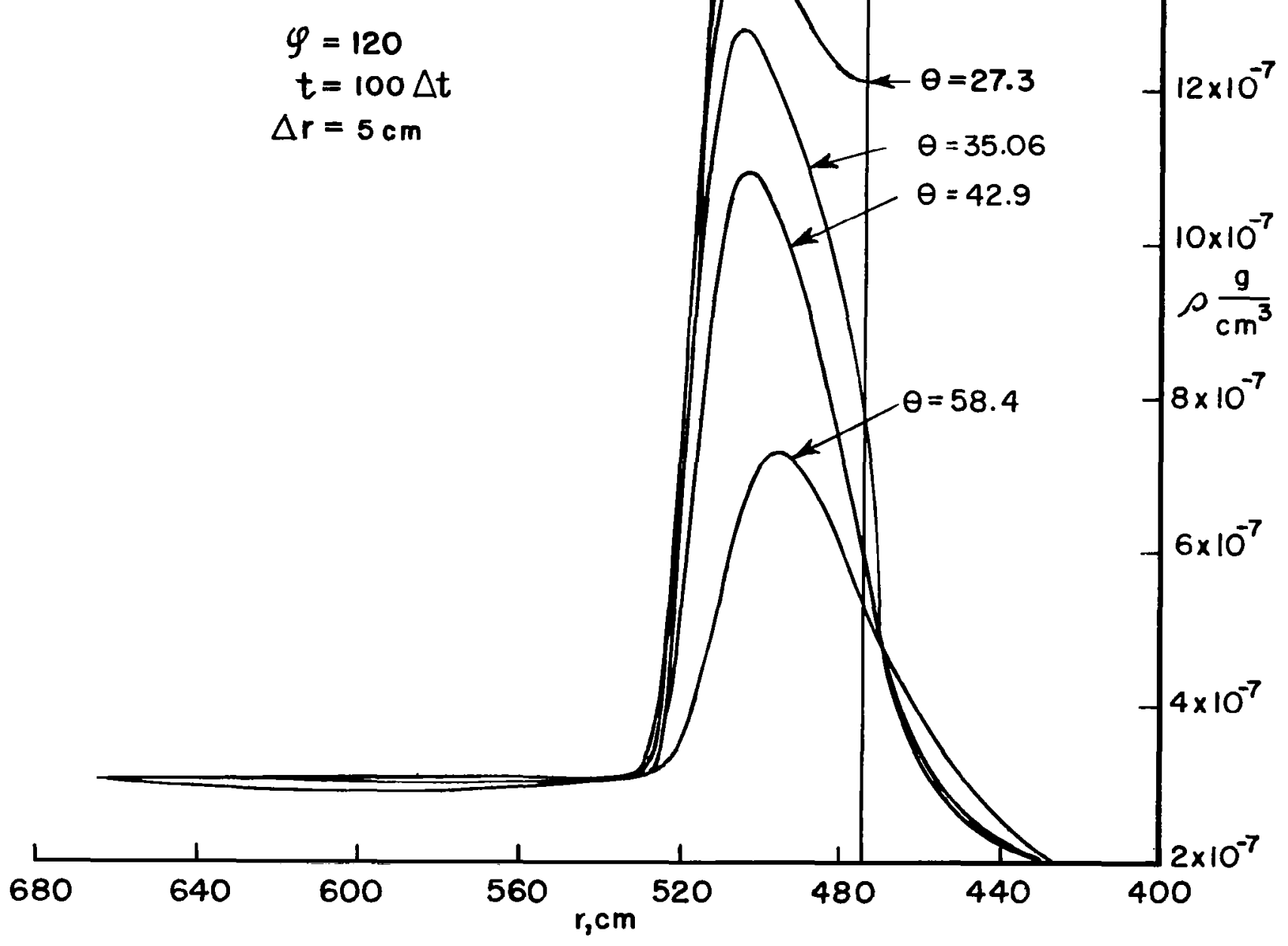

Figure 5 RADIAL DENSITY DISTRIBUTION 


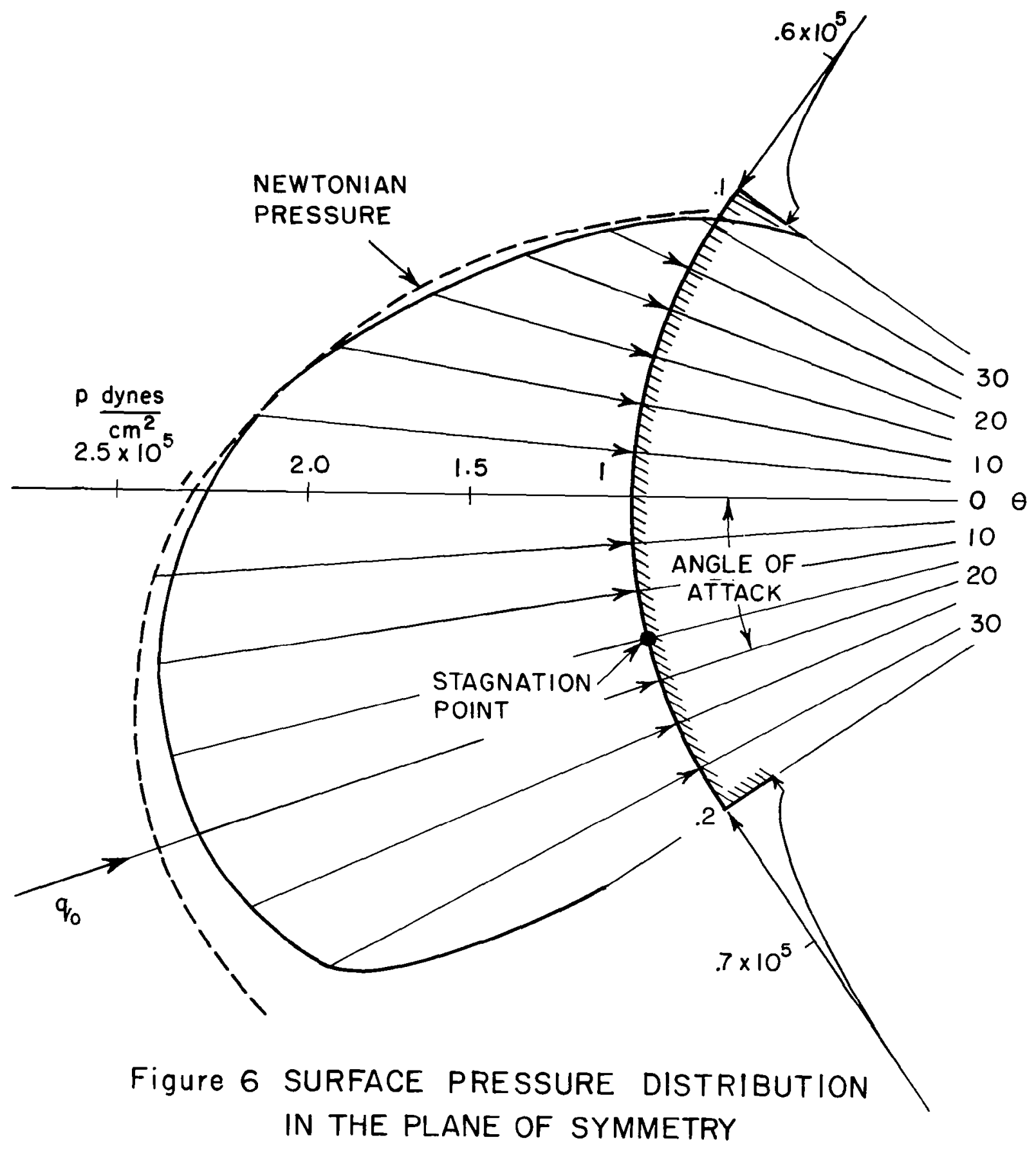




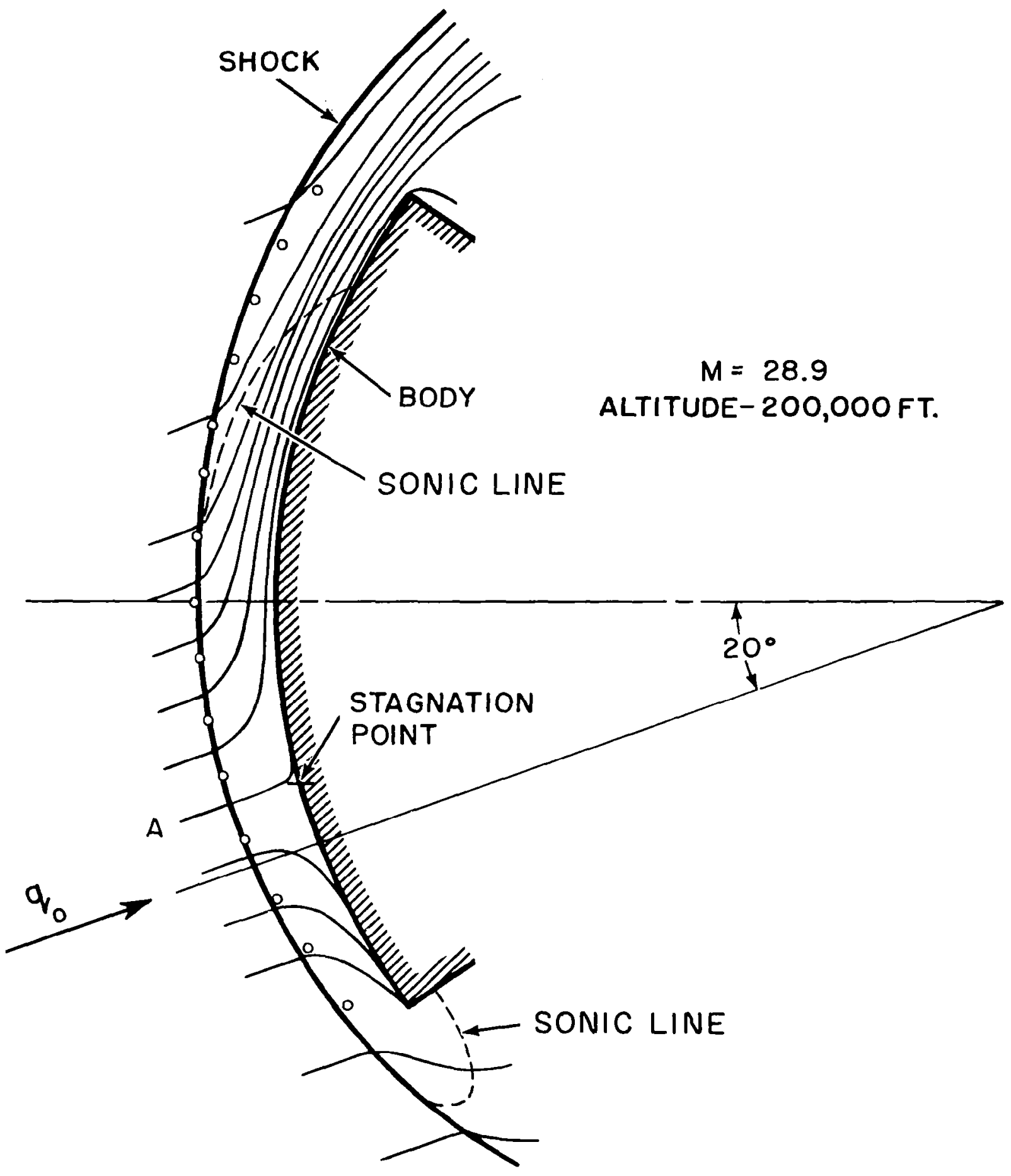

Figure 7 STREAMLINE PATTERN IN THE PLANE OF SYMMETRY 


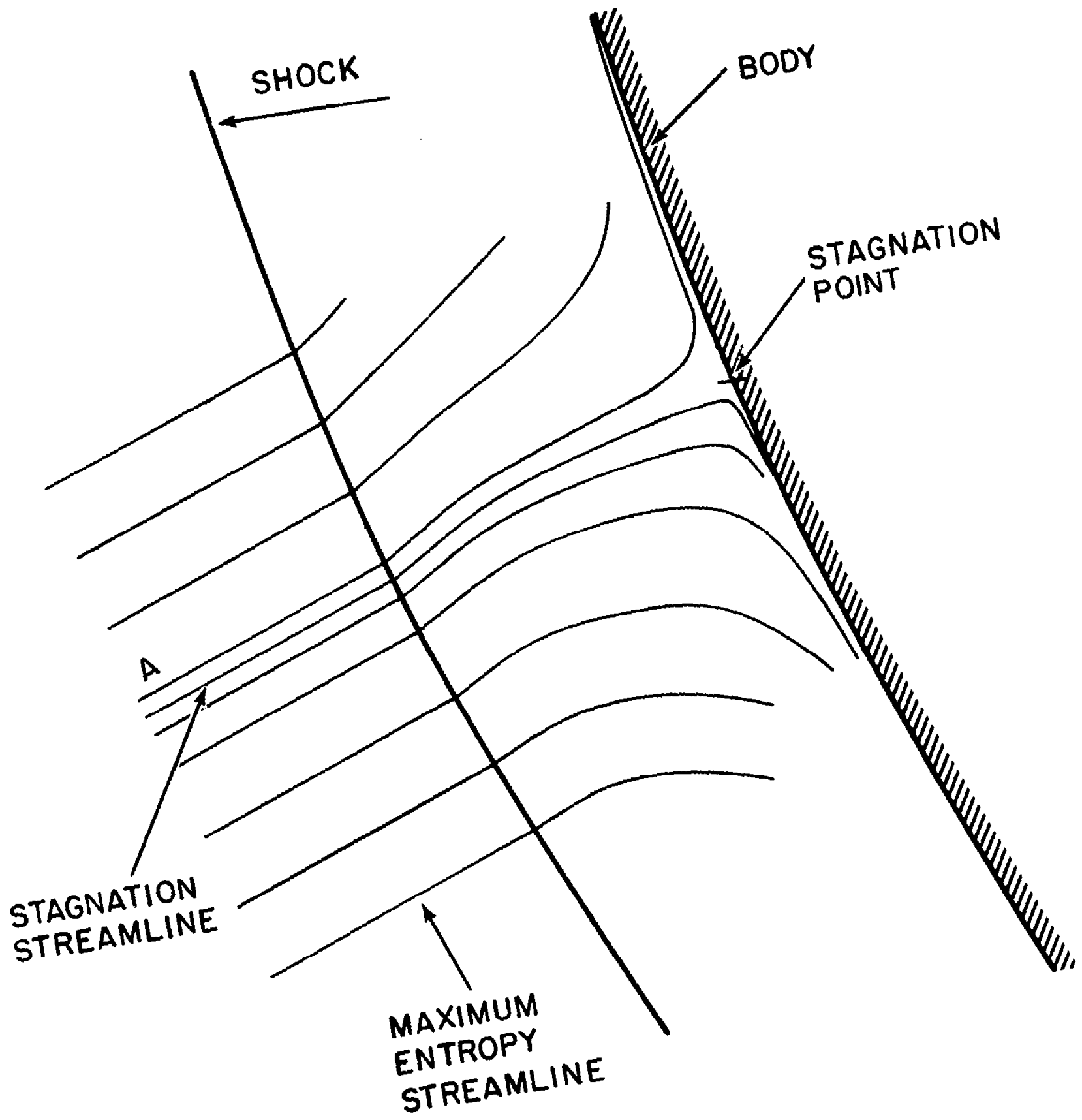

Figure 8 THE STAGNATION REGION 


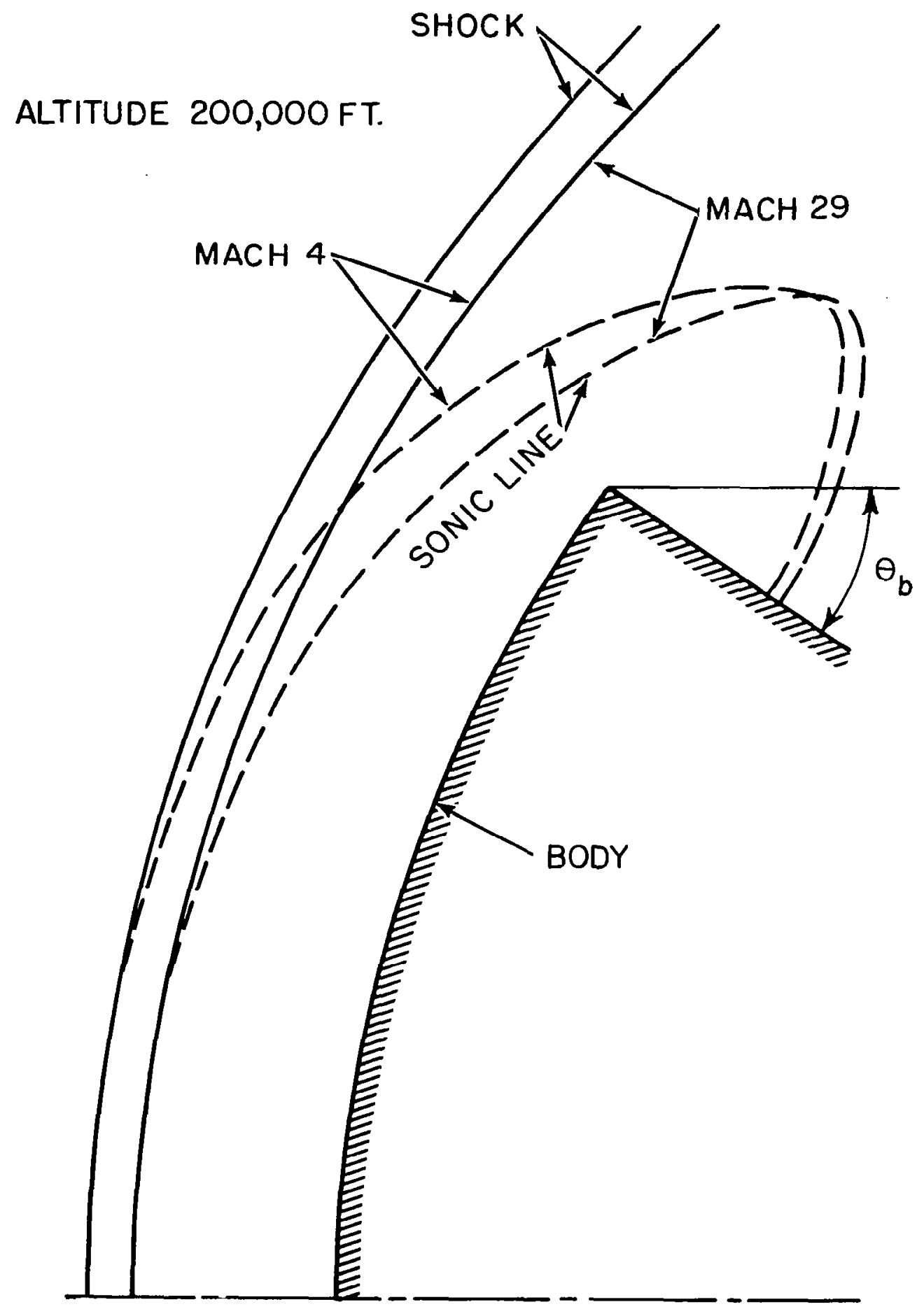

Figure 9 SHOCK AND SONIC LINE LOCATIONS FOR AXISYMMETRIC FLOW-AFTERBODY $A N G L E=35^{\circ}$ 


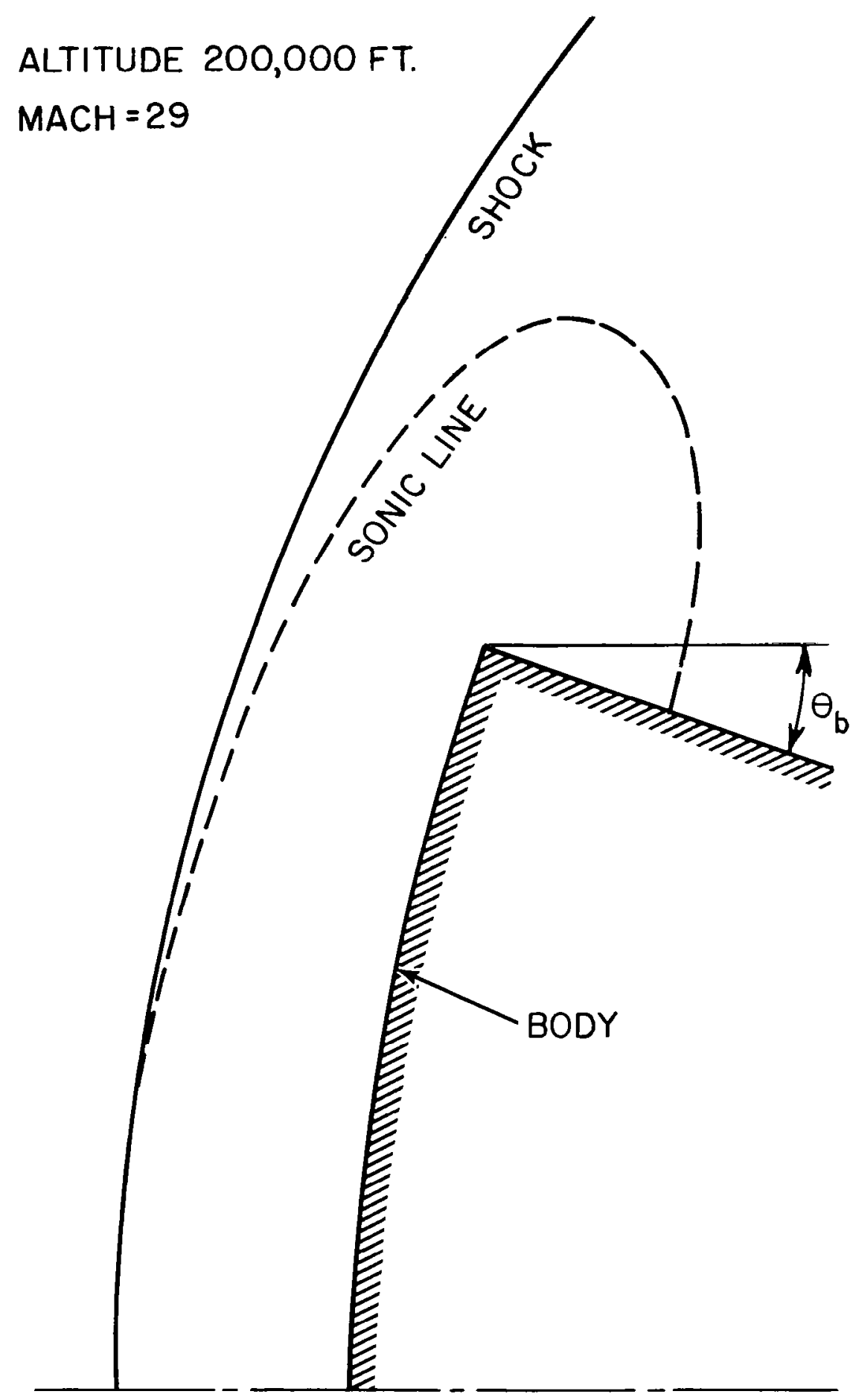

Figure IO SHOCK AND SONIC LINE LOCATIONS FOR AXISYMMETRIC FLOW - AFTERBODY ANGLE $=20^{\circ}$ 




Figure II STREAMLINE PATTERN ON THE SPHERICAL FOREBODY SURFACE 


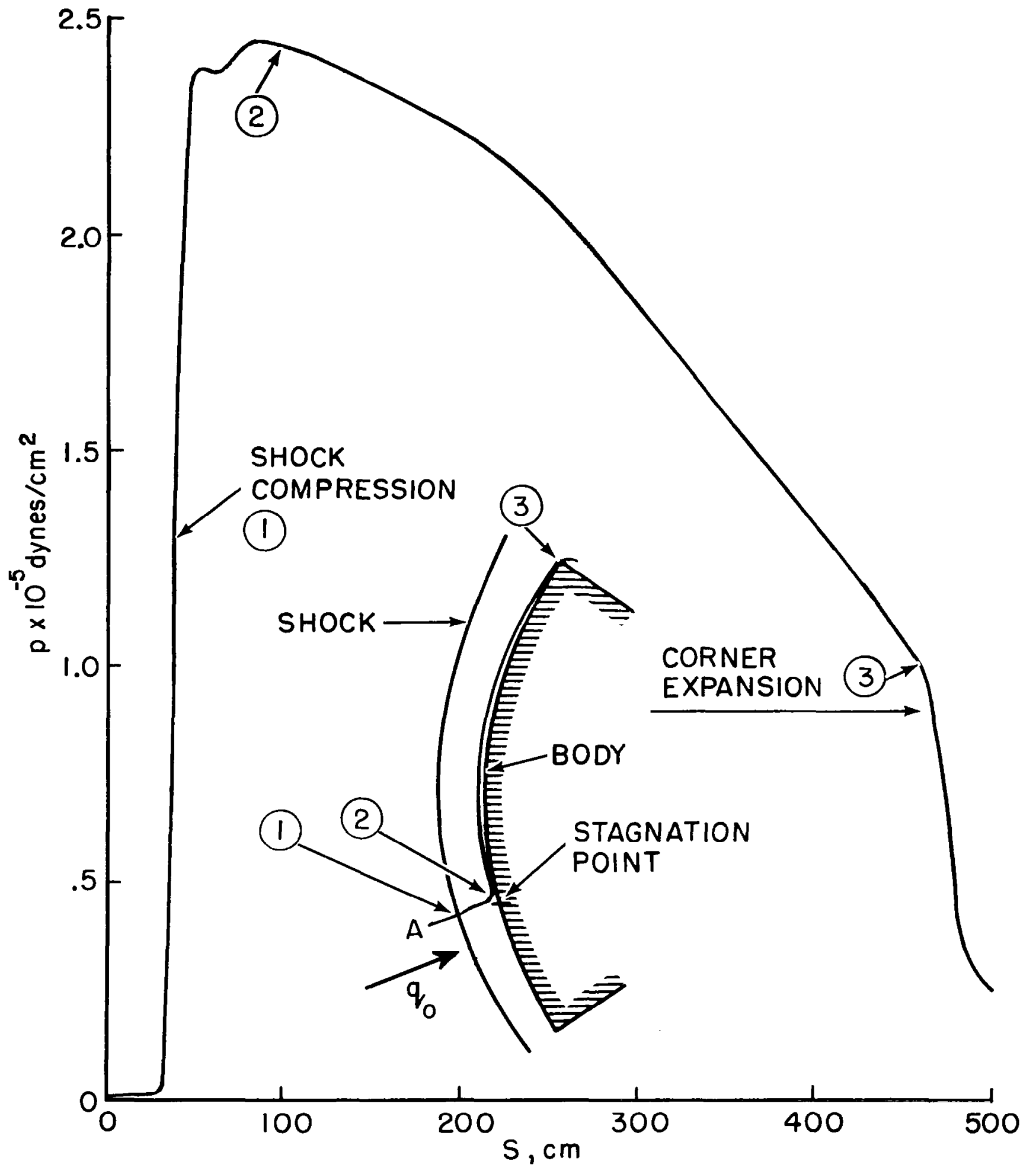

Figure 12 STREAMLINE PRESSURE DISTRIBUTION 